\title{
El no-saber en las cartografías sobre nuestro aprender como investigadores: una mirada post-cualitativa
}

Not-knowning in cartographies about our learning as researchers: a post-qualitative perspective

\author{
Carlos Canales*1 \\ canalesbcarlos@ub.edu \\ Paulo Padilla-Petry* \\ ppadillapetry@ub.edu \\ LUispe GutiérRez** \\ luispe.gutierrez@gmail.com \\ * Universitat de Barcelona, España \\ ** Universidad del País Vasco, España
}

\section{Resumen:}

Partimos de un proyecto sobre cómo aprenden los docentes a partir de la creación de cartografías sobre sus escenarios de aprendizaje, los tránsitos y la relación dentro-fuera de la escuela/instituto. Antes de empezar nuestra investigación con el profesorado de educación infantil, primaria y secundaria, nuestro grupo de investigación empezó construyendo sus propias cartografías. Éstas son entendidas como una metodología de investigación narrativa y artística que nos ha permitido explorar intersticios, desplazamientos, trayectorias inestables, maneras de saber, montajes y enredos a través de los cuales los profesores siguen sus caminos de aprendizaje. El proceso de construcción de la cartografía

\begin{abstract}
:
The starting point of this article is a project about how teachers learn using cartographies to represent their learning scenarios, their transits and the relations of schools with what is outside them. Before beginning our research with kindergarten, primary and secondary school teachers, our group built their own cartographies. They are understood as an investigation methodology that is both narrative and artistic and allows us to explore interstices, displacements, unstable trajectories, forms of knowing, assemblages and plots which teachers use for learning. The process of building cartographies about how, where and why we learn how to be researchers made us change the focus of
\end{abstract}

\section{Dirección para correspondencia (correspondence address):}

Carlos Canales. Universidad de Barcelona. Departamento de Artes Visuales y Diseño. Unidad de Pedagogías Culturales. Facultad de Bellas Artes. Pau Gargallo, 4. 08028 Barcelona (España). 
El no-saber en las cartografías sobre nuestro aprender como investigadores: una mirada post-cualitativa

Carlos Canales, Paulo Padilla-Petry y Luispe Gutiérrez

sobre cómo, dónde y porqué aprendemos a ser investigadores nos obligó a cambiar el foco de la investigación. También nos obligó a cambiar la lente con la que miramos y el ángulo desde el que observamos. Investigarse para posteriormente investigar partiendo de metodologías artísticas: un paradigma que coloca al investigador en una posición de inseguridad y fragilidad. En el artículo partimos desde una posición de no-saber para realizar una revisión y estudio sobre la construcción de nuestras propias cartografías. Por otro lado, intentamos dar cuenta de muestras del proceso de cuestionamiento epistemológico, ontológico, ético y metodológico que parten de nuestro acercamiento a postulados de investigación post-cualitativos.

\section{Palabras clave:}

Post-cualitativo; cartografías; aprender; investigar con métodos artísticos. our research. It also made us change the lens through which we look and the angle from which we observe. We did research about ourselves with a view to inquiring through artistic methods. This paradigm puts the researcher in a position of fragility and insecurity. In the article we situated ourselves in an unknown position with a view to reviewing and studying the process of building our cartographies. On the other hand, we tried to explain samples of our epistemological, ontological, ethical and methodological processes which stemmed from post-qualitative investigation assumptions.

\section{Key words:}

Post-qualitative; cartographies; learning; research with artistic methods.

\section{Résumé:}

Nous partons d'un projet sur la façon dont les enseignants apprennent à partir de la création de cartographies sur leurs scénarios d'apprentissage, les transits et leurs relations au sein de l'école / lycée. Avant de commencer notre recherche avec les enseignants de la petite enfance, de l'enseignement primaire et secondaire, notre groupe de recherche a commencé à élaborer ses propres cartographies. Celles-ci sont comprises comme une méthodologie de recherche narrative et artistique qui nous a permis d'explorer des interstices, des déplacements, des trajectoires instables, des façons de savoir, des assemblages et des enchevêtrements à travers lesquels les enseignants suivent leur parcours d'apprentissage. Le processus de construction de la cartographie sur comment, où et pourquoi nous apprenons à être des chercheurs nous a obligés à changer l'objet de la recherche. Cela nous oblige également à changer la lentille avec laquelle nous regardons et l'angle sous lequel nous observons. Enquêter pour rechercher plus tard selon des méthodologies artistiques: un paradigme qui place le chercheur dans une position d'insécurité et de fragilité. Dans l'article, nous sommes partis d'une position de nonsavoir pour mener un examen et une étude sur la construction de nos cartographies. D'autre part, nous essayons de rendre compte d'échantillons du processus de questionnement épistémologique, ontologique, éthique et méthodologique qui partent de notre approche des postulats de recherche post-qualitative.

\section{Mots clés:}

Post-qualitatif; cartographies; apprendre; enquêter avec des méthodes artistiques.

Fecha de recepción: 22-11-2018

Fecha de aceptación: 29-5-2019 


\section{Introducción}

En el proyecto de investigación (APREN-DO: Cómo aprenden los docentes: implicaciones educativas y retos para afrontar el cambio social - Ministerio de Economía y Competitividad / EDU2015-70912-C2-1-R) sobre cómo aprenden los docentes a partir de la creación de cartografías sobre sus escenarios de aprendizaje, los tránsitos y la relación dentro-fuera de la escuela y/o el instituto, partimos de una posición de no-saber (Atkinson, 2011) ante una nueva forma de conocer esta realidad e investigarla.

Sin embargo, antes de empezar la investigación con el profesorado de educación infantil, primaria y secundaria, nuestro grupo de investigación realizó, como ya ha ensayado en anteriores proyectos, las estrategias y los métodos que luego se utilizarían para relacionarnos con el profesorado. Tratamos de realizar primero nosotros lo que posteriormente deberían realizar los docentes. Así, nuestro grupo empezó diseñando y construyendo sus propias cartografías de aprendizaje como docentesinvestigadores en la universidad, entendiéndolas como una metodología de investigación narrativa y artística que nos permitiese explorar intersticios, desplazamientos, trayectorias inestables, maneras de saber, montajes y enredos a través de los cuales los profesores siguen sus caminos de aprendizaje (Liebman \& Paulston, 1994; Ruitenberg, 2007; Ulmer \& Koro-Ljungberg, 2015).

Esta primera cartografía sobre cómo, dónde y porqué aprendemos a ser investigadores nos obligó a cambiar el foco de la investigación que Ilevamos a cabo posteriormente. Pero no solo el foco, sino también la lente con la que miramos y el ángulo desde el que observamos quedaron afectados y trastocados durante el proceso. Investigarse para posteriormente investigar, partiendo además de metodologías artísticas, es la perspectiva que se abre y desarrolla en nuestro proyecto. Un enfoque que coloca al investigador en una posición de inseguridad y fragilidad ante lo desconocido. El estudio inicial, la prueba piloto sobre nosotros mismos, la construcción de nuestras cartografías implicó y nos obligó a elaborar cuestionamientos epistemológicos, ontológicos, metodológicos y éticos del proceso llevado a cabo, que partían de un replanteamiento desde perspectivas teóricas de investigación e indagación post-cualitativas.

En este artículo intentamos dar cuenta de una revisión de esta experiencia inicial a partir de alguno de los conceptos por los que estamos 
El no-saber en las cartografías sobre nuestro aprender como investigadores: una mirada post-cualitativa

Carlos Canales, Paulo Padilla-Petry y Luispe Gutiérrez

transitando, tratando la elaboración de las cartografías desde una mirada que centra su atención en la posición de no-saber, que nos sirve para nuestra revisión y mirada posterior con los docentes y que resulta afectada por los planteamientos post-cualitativos.

\section{Algunos conceptos que nos permiten pensar: marco teórico}

Los procesos y experiencias que describimos se producen en un momento temporal en el que ambos grupos de investigación, Esbrina y Elkarrikertuz, empiezan a interesarse sobre posiciones y planteamientos derivados de las perspectivas post-cualitativas de investigación, los nuevos materialismos, las nuevas ontologías y los nuevos empirismos, cuestionando así cómo debe plantearse el proyecto y cómo abordar la propia investigación. El giro ontológico, epistemológico y ético que parte de las lecturas (Barad, 2007a; Barad, 2007b; Barad, 2003; Braidotti, 2016; Haraway, 1988; Lather, 2016; St. Pierre, 2016; St. Pierre, Jackson \& Mazzei, 2016; De Freitas, 2016), generan en el grupo tensiones ante las nuevas formas de entender la realidad y el conocimiento que se transforman en momentos de duda, fragilidad y no-saber. No obstante, y a su vez, son estos mismos planteamientos y esta posición o revisión de la noción de no-saber la que nos están permitiendo abordar la investigación y entenderla desde una nueva perspectiva y mirada.

No es la finalidad de este artículo abordar una genealogía que describa las tensiones del giro ontológico, epistemológico y ético que supone el situarse bajo una nueva perspectiva, tarea que ya se realizará en otros artículos. Pero sí abordar cuál es nuestra experiencia desde una posición de fragilidad ante la resolución de un problema que era para nosotros totalmente desconocido: indagar/investigar (generar conocimiento) sobre cómo aprendemos como investigadores-educadores partiendo de la elaboración de una cartografía.

Para abordar y entender a qué nos referimos cuando describimos el proceso llevado a cabo, nos sirve de ayuda el concepto que explora Denis Atkinson (2015) tratando de establecer una pedagogía basada en el no-saber. Atkinson pretende construir una nueva opción que no esté basada en la idea esencialista que entiende al profesor y al alumno como entidades independientes que tratan de generar saberes a través de la relación establecida entre ambos. Esta posición pedagógica esen- 
cialista puede definirse como aquella en que es el profesor el que posee el conocimiento que debe transmitir, y que son los alumnos los que deben adquirirlo según sus habilidades y capacidades. Habilidades y capacidades que pueden medirse y cuantificarse a través de pruebas que deben superar los estudiantes, quienes, además, deben demostrar cómo de eficientes son a la hora de obtener y reproducir los aprendizajes adquiridos. En esta posición de transmisión del conocimiento unidireccional, los estudiantes están subordinados al docente a la hora de obtener la información valiosa. Hay una jerarquía clara dentro de la relación. Estas entidades (profesor-alumno) son entendidas como preexistentes a la relación pedagógica, y el conocimiento sólo queda supeditado a la capacidad del profesor de transmitir y a la capacidad del alumno por adquirirlo. No se genera de la relación de intercambio, sino que existe también, de forma esencial, como objeto que puede transferirse sin que cambie su esencia.

En nuestro caso, las entidades que entran en juego son otras, como también lo es la forma en que se entienden los saberes, como fruto de la propia relación. El investigador (sujeto) elabora y se relaciona con una producción visual (metodología-objeto) que genera un conocimiento al desarrollar este proceso. Éste se produce de la relación entre la metodología (la producción visual), el investigador (sujeto) y la producción visual de la cartografía (metodología - objeto material). Desde un punto de vista esencialista, en nuestro caso, son las entidades sujeto-objeto/metodología las que son vistas como entidades separadas que generan un saber en su relación. Por tanto, los saberes están supeditado al investigador, que es capaz de extraerlos a través de la relación con una metodología u objeto visual. Pero, aunque esta separación ontológica entre entidades puede parecer que facilita el entendimiento de la relación pedagógica y/o nuestra investigación, para nosotros, no es del todo así.

El primer motivo es que separar las entidades de forma individual y de este modo supone configurarlas como entidades preexistentes. Es decir, que tienen una serie de atributos que les son necesarios para conformar su identidad y función. Sin estos atributos, la entidad no existe como tal. El segundo motivo es que presupone una jerarquía dentro de la relación: quién posee, cómo obtiene y cómo puede entregar los saberes. Se presupone que el conocimiento está supeditado al ser humano que lo posee y entrega, o supeditado a la persona que lo recibe e interioriza, como si fuese una entidad inerte que pudiese ser obtenida o entregada, dada o 
El no-saber en las cartografías sobre nuestro aprender como investigadores: una mirada post-cualitativa

Carlos Canales, Paulo Padilla-Petry y luispe Gutiérrez

recibida. Es pensar que éste no se genera, precisamente, de la relación entre sujetos o en la relación de sujetos con otras entidades no-humanas (objetos materiales) o metodológicas (cartografías). El tercer motivo tiene que ver con cómo la producción visual, o el hecho en sí de producir visualmente, forma parte de la relación que genera y, por tanto, tiene tanto o más peso en la relación que el propio investigador. Es entidad en sí misma, no quedando supeditada una entidad a la otra. Para entender la importancia de las producciones cartográficas en relación a nuestro proyecto, así como ampliar planteamientos post-qualitativos en relación con las cartografías, sugerimos revisar los trabajos de Guattari (2000); Guattari y Rolnik (2006), Masny (2013) y Ulmer \& Koro-Ljungberg (2015).

Volviendo a nuestro desarrollo, podemos entender que es tanto en la relación pedagógica, así como en la relación de los sujetos con los objetos, donde se encuentra lo interesante y lo valioso, y no tanto en las entidades en sí mismas. Sin relación, no hay entidades. Un investigador se convierte en investigador cuando se relaciona con lo investigado, y lo investigado se convierte en tal cuando un investigador lo investiga. Lo mismo podríamos decir de lo metodológico: no hay condición de metodología hasta que un investigador no la activa y no hay investigador hasta que no es activado por una metodología. Aunque parece confuso, entendemos que es en la relación entre entidades cuando el conocimiento deviene como tal. Este cambio ontológico es el que nos ayuda a entender y abordar nuestro objeto de estudio. No obstante, es esta posición la que genera situaciones de tensión (e incomprensión) entre los propios investigadores.

Para entender y explicar esta posición ética dentro de la investigación (y entendemos como ética por el hecho de no configurar las entidades de forma preexistente y esencialista sino una vez establecida la relación), Dennis Atkinson (2015) utiliza el término de intra-action ('intra-acción') de Karen Barad (2003; 2007b) como concepto clave que le permite proponer una nueva forma de entender la práctica pedagógica desde una posición de no-saber y desde la idea de los subjects-yet-to-come ('sujetos por devenir'). La noción propuesta por Karen Barad (2003) difiere del término inter-action ('interacción') que "presupone la existencia previa de entidades independientes" (p. 815), como acabamos de explicar, y representa un cambio profundo sobre cómo entendemos la realidad desde posiciones no esencialistas. Como hemos comentado, podemos entender de su noción que las entidades (sujeto-objeto-conocimiento) no son 
independientes, sino que es la serie de relaciones que hacen que estas entidades emerjan, que devengan como tales. Para Barad, la "existencia no es un hecho individual. Los individuos no preexisten a sus interacciones; más bien son los individuos los que emergen a través de y como parte de su entangled intra-relating ('enredo intra-relacionado')" (2007b, p. 9). Llevándolo al terreno de lo pedagógico, Atkinson propone que, si nos basamos en esta concepción de la realidad, debemos situarnos y basarnos, en cierta medida, "en la idea del no-saber, porque es sólo mediante las intra-acciones de la relación pedagógica que lo conocido deviene en conocido. Es decir, es solo como una consecuencia de las intra-relaciones que el alumno, el educador y el conocimiento pueden emerger como tales" (2015, p. 45). O, situándonos en nuestro caso, es sólo a través de una posición ética de no-saber que los sujetos (investigadores), los objetos (cartografías) y el conocimiento (cómo aprenden los docentes) pueden emerger.

Sin duda, todo ello tiene que ver con el giro ontológico de los nuevos materialismos, que nos invita a ver la realidad desde una estructura no jerárquica de la misma. Maggie MacLure (2013, p. 659), cita el término que utilizan algunos autores (De Landa, 2002; Hultman \& Lenz Taguchi, 2010) para definir esta nueva lógica como flattened ('aplana$\mathrm{da}^{\prime}$ ). Además, otros autores han transitado por posiciones parecidas bajo conceptos como assemblage ('ensamblaje') (Deleuze \& Guattari, 2004); entaglement ('enredo') (Barad, 2007b); o la misma teoría sobre el actor red ('red de actores') de Bruno Latour (2007), entre otros.

\section{Sobre cómo se desarrolló el proceso: marco empírico}

Como se ha indicado, nuestro equipo de investigación tiene como uno de sus principios metodológicos y éticos realizar siempre una primera recogida de datos con nosotros mismos. Esto supone que antes de relacionarnos con los participantes, ocupamos nosotros una posición similar a la suya en nuestra investigación. Para conseguir esto, realizamos las mismas tareas previstas que se pedirán realizar a los participantes. Así, en investigaciones previas, hemos escrito sobre nuestra relación con el saber (Padilla \& Hernández, 2013; Gutiérrez, Correa, Jiménez \& Ibáñez, 2009) o sobre nuestra vida en la universidad (Padilla, Hernández \& Creus, 2014), por citar dos ejemplos. Esa práctica inicial que repetimos 
El no-saber en las cartografías sobre nuestro aprender como investigadores: una mirada post-cualitativa

Carlos Canales, Paulo Padilla-Petry y Luispe Gutiérrez

en toda investigación nos permite reflexionar sobre los objetivos y los métodos desde otra posición. También nos permite acercarnos al problema de estudio desde otra perspectiva. Desde el punto de vista ético, además, garantiza que, antes de nada, nos enfrentemos a nuestro no-saber.

Así, cada miembro del grupo de investigación construyó su cartografía sobre cómo aprendió a ser investigador y profesor universitario. Para eso, el equipo pasó dos días reunido en un aula de la Universidad de Barcelona que habitamos a modo de taller elaborando nuestras cartografías. La mayoría de nosotros (12 de 18) optó por el formato digital preparando sus cartografías a través de herramientas digitales que permitieran dibujar, insertar imágenes y texto. Los otros 6 investigadores optaron por cartografías analógicas dibujando, escribiendo y pegando imágenes sobre cartulinas. El hecho de estar reunidos en un mismo espacio propició el intercambio de ideas y experiencias. Igualmente, la presencia de estudiantes de doctorado y de máster de la Facultad de Bellas Artes posibilitó cambios a la hora de formalizar o graficar las cartografías.

Una vez finalizadas las cartografías, y en los días subsiguientes, cada investigador escribió un texto explicando el proceso de construcción de las cartografías y reflexionando sobre las decisiones tomadas respecto a la elaboración de la cartografía. El presente artículo se basa en la extracción y análisis de esos textos.

\section{Qué hemos encontrado sobre el no saber: aportaciones}

Nuestros textos sobre nuestras cartografías han puesto en evidencia diferentes formas de nuestro no-saber. Como venimos anticipando desde el inicio del artículo, es sólo a partir de las relaciones (intra-acciones) entre los objetos y los sujetos que pueden entenderse tanto las cartografías como los propios investigadores. Es por eso por lo que pretendemos dar cuenta de estas relaciones, caracterizadas por una posición de incertidumbre, incomodidad y sorpresa, de no-saber, pero con un carácter muy claro y una implicación que pretendía extraer conocimientos del proceso de elaboración de nuestras cartografías.

La primera manifestación que rescatamos fue la incomodidad generada por la tarea que nos exigía una representación visual de nuestro aprendizaje. Como investigadores del aprendizaje de jóvenes y del profesorado, ya hemos escrito sobre cómo se aprende y cómo aprendemos 
(Hernández (ed.), 2017; Sancho \& Hernández (ed.), 2014; Gutiérrez, 2014; Correa, Martínez \& Gutiérrez, 2013; Martínez \& Gutiérrez, 2013). Sin embargo, el giro espacial (Jones et al., 2016) que supone construir una cartografía nos dejó sin dos recursos muy utilizados en nuestro contexto: el discurso o la narrativa. Por un lado, no podíamos explicarnos con tantas palabras escritas como nos gustaría y por otro nos era más difícil (o nuevo) el utilizar las referencias temporales que las espaciales. La segunda manifestación de nuestro no-saber se encarnó cuando observamos el resultado que estábamos obteniendo: se generó una cierta frustración respecto a producciones que parecían no responder suficientemente a una reflexión previa y/o que no posibilitaban la representación de todo lo pretendido, como si quedasen incompletas o irrepresentadas. Una tercera manifestación del no-saber fue la inseguridad respecto a la posibilidad de legibilidad de la cartografía.

Analizaremos a continuación cada una de esas manifestaciones, para tratar de establecer alguna de las relaciones que nos permitan pensarnos como sujetos en una posición de fragilidad y no-saber y pensar a las cartografías como posibilitadoras de indagación sobre nuestros procesos de aprender, pero a su vez como objetos que nos enseñan y de los que aprendemos.

\section{De lo narrativo a lo visual}

Los dos grupos de investigación que participaron de este proceso tienen una larga tradición en investigación narrativa. A través de etnografías e historias de vida hemos descrito y narrado distintos entornos de aprendizaje y las trayectorias de jóvenes, maestras y profesores, entre otros. Para nosotros, la narrativa escrita siempre ha sido una herramienta de investigación y no una mera manera de presentar o comunicar los datos de estas. Este hecho es observable en diversos textos de los investigadores:

Mi posición como investigador siempre, desde mi tesis doctoral, se había alineado en el modelo narrativo. Ahí me siento cómodo pues, aunque escribir me cuesta, se trata de una práctica en la que encuentro cierto placer. Además, escribir y narrar me ayudan a ordenar ideas y pulir argumentos. Cartografiar, sin embargo, me sonaba a dibujar, asignatura que siempre había evitado en mi escolarización. La expresión plástica y gráfica no ha sido nunca mi expresión favorita... (Luispe) 
El no-saber en las cartografías sobre nuestro aprender como investigadores: una mirada post-cualitativa

Carlos Canales, Paulo Padilla-Petry y Luispe Gutiérrez

Desde un primer momento tuve claro que no me iba a sentir cómoda siguiendo un proceso analógico (cartulinas, lápices de colores, rotuladores...) de elaboración. No era lo mío atendiendo a mis limitaciones a la hora de expresarme "a mano alzada". (Cristina)

[...] realizar un ejercicio de plasmar mi itinerario como aprendiente o como docente en imágenes entraña especial dificultad para una persona como yo, que ha estado siempre ligada a la palabra y a la que le concede un sitio preeminente en su vida profesional y personal. (Asun)

Vemos aquí una cierta posición inicial de rechazo, o como mínimo de dificultad frente a lo nuevo y lo desconocido: el uso de una herramienta visual como método de indagación. Este hecho, además se encarna en la necesidad de tomar tiempo y no empezar a producir, en la necesidad de reflexionar previamente o dar paso a la espera antes de ponerse a representar gráficamente.

Al rememorar el momento en que los compañeros del proyecto nos encontrábamos para realizar nuestras cartografías, lo primero que me viene a la cabeza fue las dificultades que tuve para iniciarla. Un comienzo incierto para mí dado que no tenía demasiado claro qué era aquello que debía hacer. (Herraiz)

He sido una de las últimas personas (creo que aún falta alguien) en elaborar y subir al Moodle del proyecto Apren-do mi cartografía.... Los motivos.... muchos y variados, pero la explicación... muy simple... la cartografía podía esperar. (Cristina)

Consciente de que la mayoría del grupo ya ha terminado y yo todavía no he empezado, me acompaña a las reuniones la sensación de ir a remolque, pero las ideas que se me ocurren para hacer la cartografía me aburren a mí misma. No me apetece hacer una cartografía por cumplir. Y menos sobre un tema que me apasiona tanto como este. Por eso me permito esperar a entender el sentido que tiene esta cartografía para el proyecto y para mí misma. (Raquel)

Pero, no obstante, esta posición de incertidumbre o pausa inicial se asume como un reto autoimpuesto por los propios investigadores, muy implicados en aprender y dar solución al problema planteado, incluso 
Ilegando a realizarla meses después de la sesión de trabajo y dando cuenta del proceso de reflexión necesario para abordarla.

\begin{abstract}
Al no saber ni cómo hacer ni qué argumentar, mi mirada de reojo se volvió hacia los compañeros cercanos mientras escuchaba de algunos de ellos: "si yo no sé dibujar"; como si "saber dibujar" en el sentido clásico tuviese algo que ver para hacer una cartografía. (Herraiz)
\end{abstract}

¿Crear un mapa de cómo aprendo? ¿Eso se puede hacer? ¿Tendré que ponerlo todo? ¿Qué dejo fuera? ¿Es posible ser neutral? ¿Dónde miro? ¿Qué escojo? ¿Qué represento? ¿Solo lugares? ¿Y el tiempo, cómo represento el tiempo? ¿Y la alegría o la tristeza? ¿Se puede hacer un mapa de cualquier cosa? (Aingeru)

Este proceso de elaboración empieza desde la negación a realizar la cartografía. Desde junio de 2016 hasta febrero de 2017 pienso en la cartografía, pero no materializo nada. Sin embargo, eso no significa que no la hubiera empezado. ¿Acaso un dibujo empieza siempre con su primer trazo? (Raquel)

No obstante, también queremos rescatar que, opuestamente a lo que les ocurría a algunos de los investigadores en los que cambiar la narrativa escrita por una representación visual se convirtió en dar un salto al vacío, otros se sentían a gusto y disfrutando del proceso.

Cuando supe que tenía que hacer la cartografía y luego compartirla me sentí bien; quiero decir: cuando tenemos que escribir un texto siento mayor preocupación y tensión, pero una cartografía para mí es una representación visual y artística donde mostrarme en mi lenguaje, utilizando recursos con los que me siento cómoda. (Estitxu)

Cabe decir, que estos últimos en su mayoría contaban con trayectorias vinculadas a la formación artística y visual, sintiéndose a gusto, e incluso nos atreveríamos a decir reconfortados, con este tránsito.

\title{
La insuficiencia de la cartografía
}

Para algunos investigadores elaborar la cartografía confirmó un temor inicial: la insuficiencia de la cartografía como un recurso para represen- 
El no-saber en las cartografías sobre nuestro aprender como investigadores: una mirada post-cualitativa

Carlos Canales, Paulo Padilla-Petry y Luispe Gutiérrez

tar lo que se pretende. Insuficiencia o imposibilidad, podríamos decir. Como todo lenguaje, el lenguaje visual tiene límites. No todo puede ser representado visualmente. La imagen mental (el pensamiento, las ideas) es diferente de su representación física (la cartografía). Las representaciones visuales responden a unos códigos preestablecidos (en este caso visuales) y aprendidos por parte de cada uno de los investigadores, respondiendo así a unos bagajes y aprendizajes previos. Es por eso que para algunos les fue más fácil representar visualmente lo que pensaban que para otros. A su vez, y, por otro lado, también debemos tener en cuenta la imposibilidad de representar visualmente elementos que el propio lenguaje visual e inanimado no lo permite, como es el caso de los movimientos, las transformaciones, los cambios que se producen dentro de los elementos de la cartografía, por poner algunos ejemplos. Esto significa que podemos representar visualmente, mediante una metáfora, por ejemplo, un movimiento, pero no podemos hacer que la cartografía se mueva. O no, al menos, en el nivel gráfico que podíamos alcanzar. Tal como menciona Fernando al finalizar su cartografía: la cartografía fija un momento concreto y al revisarla de nuevo, los investigadores ya hemos cambiado.

Este punto me llevaría a seguir pensando sobre el proceso y el contenido de mi cartografía. Pero lo dejo ahora aquí, no sin mencionar antes que cuando entro en el espacio de investigación de Esbrina y la veo colgada en una de las paredes, siento que, a la vez que me reconozco en ella, en ese momento en el que me sentido afectado por la 'incomplitud', también siento que me voy moviendo, y que ahora hay otros lugares del aprender que me cruzan y me afectan. Pero en algún momento me he de parar. Como ahora. (Fernando)

Revisar actualmente la cartografía me lleva a tomar conciencia de los límites de la misma. (Herraiz)

También cambia nuestra relación con lo cartográfico (con la producción visual), entendiendo ésta de otro modo. Pero la cartografía elaborada sigue siendo la misma, no ha cambiado, no se mueve. 


\section{La legibilidad de la cartografía}

Otro aspecto del no-saber, este relacionado con el Otro, fue la preocupación con la legibilidad de la cartografía. La cartografía, como otros productos de nuestros proyectos de investigación sería un documento o producción pública. Así que en un primer momento las cartografías serían vistas por todo el equipo de investigación y posteriormente por cualquiera que se interesase en ellas. La supuesta o imaginada presencia de ese grupo indiferenciado y desconocido (Otro) que miraría la cartografía plantea de alguna manera una manera de enfrentarse a ella y a su producción, intentando que esta sea legible y descifrable.

Más que intentar aproximarme a la pregunta en su globalidad, decidí presentar el mapa en forma de mapa mental, con una clara jerarquía dividida en cuatro apartados. Esta presentación permite presentar la información de forma clara y concisa, aunque, como contrapartida, omite relaciones entre los apartados y también con otros aspectos de mi aprendizaje, que quedan ocultos en favor de la claridad. (Xavi)

Comencé mi diseño con la idea de que fuera lo más completo posible y que también fuera legible para "el otro". (Sandra)

Se hace un esfuerzo de utilización de códigos provenientes del diseño, la cartografía tradicional, la fotografía, el collage, entre otras estrategias, para que la cartografía sea entendible o descifrable. Este esfuerzo, no obstante, sólo funcionará bajo nuestros códigos visuales, pues es nuestra cultura visual compartida la que nos permite leer y producir las cartografías de forma que las entendamos y las podamos entender. Considerando que la cartografía es básicamente un recurso visual, hablar de su legibilidad (de leerla) puede parecer contradictorio, pero no lo es. Las cartografías deberían dar cuenta de nuestros procesos de aprendizaje para un Otro, es decir, son objetos que nos sirven como estrategias de indagación y reflexión sobre nosotros y que también nos sirven como ejemplo u objeto que comunica a otros cómo pueden usarse estos métodos, en una especie de representación de nuestros pensamientos. Es esta falta de control sobre lo que el Otro va a leer (o mirar) que nos hace posicionarnos en un lugar de duda y que nos hace intentar construir un entendimiento compartido. Este hecho hizo que cayésemos en la cuenta 
El no-saber en las cartografías sobre nuestro aprender como investigadores: una mirada post-cualitativa

Carlos Canales, Paulo Padilla-Petry y Luispe Gutiérrez

y el error absurdo o ingenuo de no pensar en que al igual que pasa con los textos, las cartografías son interpretadas (y modificadas) por los sujetos cuando las miran (o las leen).

\section{Qué pasa con nuestra posición como investigadores: discusión}

No resulta cómodo para un profesor-investigador universitario reconocerse ignorante ante una nueva manera de abordar un proyecto de investigación. Pareciera que el no-saber no tuviera lugar en la Academia; se supone, o suponemos nosotros, que un profesor-investigador universitario es antagónico a un ignorante, de manera tal, que el no-saber, nos coloca en una posición que oscila desde la fragilidad hasta la curiosidad en el mejor de los casos, pero siempre generando en nosotros cierta inquietud e inseguridad. Nos cuestiona nuestra posición y poder dentro de un sistema que creemos controlar. Además, para algunos, parte de esta inquietud estaba relacionada con el hecho de exponer su incertidumbre personal al resto de investigadores. Enfrentarse a la cartografía, entendida ésta como una nueva significación que va más allá de la definición tradicional del arte de hacer mapas, supuso un choque para todos los investigadores. Primero porque mapear no era algo a lo que estamos acostumbrados. Segundo, porque hacerlo sobre cómo aprendemos como investigadores-docentes era algo que asumíamos como complejo y difícil de descifrar, y más aún, por tanto, de plasmar o representar gráficamente. El desafío estaba, en términos de Rancière (2010) en «diseñar configuraciones nuevas de lo visible, lo decible y lo pensable; $y$, por eso mismo, un paisaje nuevo de lo posible. Pero a condición de no anticipar su sentido ni su efecto» (p. 105).

De todos modos, esta ignorancia, este no-saber... ¿De qué no-saber se trata? No es un no-saber socrático, pues Sócrates sabía, aunque dijera que solo sabía que no sabía nada. El maestro de Platón se coloca en la ignorancia, pero no es ignorante. Al revés, a través de la mayéutica va desmontando todas las afirmaciones y teorías de los "sabios" de su época.

Tampoco es un no-saber al estilo del «Maestro ignorante» de Jaques Rancière (2003), donde se describe una didáctica que se opone al método clásico y que se funda en la capacidad de aprender por uno mismo más que en la transferencia del saber del maestro al alumno. Este sí que 
no sabe, pero también es cierto que no se juega nada en el acto de nosaber. De hecho, es la voluntad de sus alumnos y el método que siguen éstos los que salen triunfantes de la experiencia. El maestro no aprende, aunque sí que es cierto que el hecho de colocarse en una posición débil resulta un estímulo para sus alumnos, que tienen que tomar una actitud más activa y hacerse cargo de su aprendizaje.

Quizás, la experiencia más parecida a la que hemos vivido en este proyecto es el no-saber de alguien, en el sentido más lacaniano del término: lograr una ignorancia fecunda (Lacan, 2010: 310). Y decimos fecunda porque, finalmente, todos hicimos nuestra cartografía, es decir, acabamos atreviéndonos a atravesar ese no-saber, al igual que un analizante se atreve a tumbarse en el diván y empezar a indagar sobre su no-saber inconsciente. Recuperamos así la posición de no-saber de Atkinson para una pedagogía que parta de la idea de intra-acción, donde son las relaciones las que nos conforman como sujetos. Pues es en la relación con las cartografías que devenimos como sujetos investigadores, que devienen éstas como tales, y que podemos rescatar un conocimiento de todo ello. Atreverse a atravesar (el Rubicón de la cartografía) fue el primer efecto directo que tuvo la investigación en nosotros y en la misma. Atrevimiento que estuvo claramente marcado por una posición ética y situada, diferente en cada caso, subjetiva y, por lo tanto, personal e intransferible.

\section{Qué rescatamos de todo ello: conclusiones}

A modo de conclusión, tratamos de definir cómo a través de una posición de no-saber estamos configurando las entidades de la relación: los investigadores, las cartografías y el conocimiento generado. Partiendo de la noción de intra-action, vemos cómo las cartografías (por la relación que hemos tenido con ellas) nos están definiendo y cómo las estamos definiendo nosotros a ellas. Así, nuestra relación nos ha constituido como investigadores que tienen una clara definición de lo que quieren alcanzar, que tienen muchas dudas en el camino, ciertas fragilidades y dificultades a la hora de enfrentarse a un nuevo lenguaje visual, que reflexionan y se toman el tiempo para pensar antes de producir, que utilizan ese no-saber como potenciador de su propio desarrollo... Por otro lado, las cartografías se convierten en un objeto que nos invita a 
El no-saber en las cartografías sobre nuestro aprender como investigadores: una mirada post-cualitativa

Carlos Canales, Paulo Padilla-Petry y Luispe Gutiérrez

reflexionar, limitado por su propia materialidad en el sentido que no puede representarlo todo, que se deja cosas fuera, que es incompleto, que tiene límites irrepresentables, que fija un momento, pero a su vez que abre posibilidades de exploración en el tránsito hacia la representación visual de las ideas, que es móvil y cambiante, que deviene de la relación con el sujeto, cuestionándolo y haciendo que piense y se exprese de otro modo. Esta relación, además de constituirnos como sujetos y constituir las cartografías como objetos que forman parte de la relación como agentes activos, también han generado ciertos conocimientos situados, tomando el término de Donna Haraway (1988), que van más allá del aprendizaje de la utilización de una herramienta o un método visual, que trastocan el propio sentido de lo que es investigar, de lo que es la realidad, o mejor dicho, de cómo nos posicionamos frente a ésta como sujetos que no-saben y que se dejan sorprender y afectar por la relación. Así, es la relación y la posición de no-saber la que nos ha permitido descubrir una nueva mirada y posición, que nos acerca hacia perspectivas post-cualitativas que nos han permitido hacer una revisión o revisitar nuestra experiencia.

\section{Referencias}

Atkinson, D. (2011). Art, Equality and Learning: Pedagogies Against the State. Rotterdam: Sense Publishers.

Atkinson, D. (2015). The adventure of pedagogy, learning and the not-known. Subjectivity, $8(1), 43-56$.

Barad, K. (2003) Posthumanist performativity: Toward an understanding of how mater comes to matter. Signs: Journal of Women in Culture and Society, 28(3), 801-831.

Barad, K (2007a). Chapter 2. Diffractions: Differences, Contingencies and entanglements that matter. En Barad, K. (Ed.), Meeting the Universe Halfway: Quantum Physics and the Entanglement of Matter and Meaning (pp. 71-96). Durham: Duke University Press.

Barad, K. (2007b) Meeting the Universe Halfway: Quantum Physics and the Entanglement of Matter and Meaning. Durham and London: Duke University Press.

Braidotti, R. (2015). Posthumanismo: La vida más allá del individuo. En Braidotti, R. (Ed.), Lo Posthumano (pp. 25-70). Barcelona: Gedisa.

Correa, J.M.; Martinez, M.A. \& Gutiérrez, L.P. (2013): Between the real school and the ideal school: another step in building a teaching identity. Educational Review, 66(4), 447-464.

De Freitas, E. (2016). The New Empiricism of the Fractal Fold: Rethinking Monadology in Digital Times. Cultural Studies Critical Methodologies, 16(2), 224-234.

DeLanda, M. (2002). Intensive science and virtual philosophy. London: Continuum. 
El no-saber en las cartografías sobre nuestro aprender como investigadores: una mirada post-cualitativa Carlos Canales, Paulo Padilla-Petry y Luispe Gutiérrez

Deleuze, G. \& Guattari, F. (2004). A thousand plateaus. (B. Massumi, Trans.). London: Continuum.

Guattari, F. (2000). Cartografías Esquizoanalíticas. Buenos Aires: Ediciones Manantial.

Guattari, F.; Rolnik, S. (2006). Micropolítica. Cartografías del deseo. Madrid: Traficantes de Sueños.

Gutiérrez, L.P. (2014). La investigación narrativa en el prácticum de magisterio a través de micro-relatos docentes: el relato de Montse. Tendencias Pedagógicas, 24, 23-40.

Gutiérrez, L.P.; Correa, J.M.; Jiménez de Aberasturi, E. \& Ibáñez, A. (2009). El modelo reflexivo en la formación de maestros y el pensamiento narrativo: estudio de un caso de innovación educativa en el Practicum de Magisterio. Revista de Educación, 350, 493-505.

Haraway, D. (1988). Situated Knowledges: The Science Question in Feminism and the Privilege of Partial Perspective. Feminist Studies, 14(3), 575-599.

Hernández, F. (2017) (Ed.). ¡Y luego dicen que la escuela pública no funciona! Barcelona: Octaedro.

Hultman, K. \& Lenz Taguchi, H. (2010). Challenging anthropocentric analysis of visual data: A relational materialist methodological approach to educational research. International Journal of Qualitative Studies in Education, 23, 525-542.

Jones, S., Thiel, J. J., Dávila, D., Pittard, E., Woglom, J. F., Zhou, X., Brown, T., \& Snow, M. (2016). Childhood geographies and spatial justice: making sense of place and space-making as political acts in education. American Educational Research Journal, 53(4), 1126-1158.

Lacan, J. (2010). El Seminario. Libro 2. Buenos Aires: Paidós.

Lather, P. (2016). Top Ten+ List: (Re)thinking Ontology in (Post)Qualitative Research. Cultural Studies, 16(2), 125-131.

Latour, B. (2007). Reassembling the social: An introduction to actor network theory. Oxford: Oxford University Press.

Liebman, M. \& Paulston, R. (1994). Social Cartography: a new methodology for comparative studies. Compare, 24(3), 233-245.

MacLure, M. (2013). Researching without representation? Language and materiality in post-qualitative methodology. International Journal of Qualitative Studies in Education, 26(6), 658-667.

Masny, D. (2013)(Ed.). Cartographies of Becoming in Education. A Deleuze-Guattari Perspective. Rotterdam: Sense Publishers.

Martínez, S. \& Gutiérrez, L.P. (2013): La elección de ser docente. Cuadernos de Pedagogía, 436, 22-25.

Padilla-Petry, P., Hernández-Hernández, F. \& Creus, A. (2014). Let's begin with ourselves: attempting resonance responses in the exchange of researchers' professional autobiographies. Journal of Curriculum Studies, 46 (6), 819-838

Padilla-Petry, P. \& Hernández-Hernández, F. (2013). Ideais e alteridade em uma pesquisa sobre a relação dos jovens com o saber. Estilos da clínica, 18(1), 14-33.

Ranciere, J. (2010). El espectador emancipado. Buenos Aires: Ediciones Manantial.

Ranciere, J. (2003). El maestro ignorante: cinco lecciones sobre la emancipación inte- 
El no-saber en las cartografías sobre nuestro aprender como investigadores: una mirada post-cualitativa

Carlos Canales, Paulo Padilla-Petry y Luispe Gutiérrez

lectual (Núria Estrach, trad.). Barcelona: Editorial Laertes. (Obra original publicada en 1987).

Ruitenberg, C. W. (2007). Here be dragons: Exploring Cartography in Educational Theory and Research. Complicity. International journal of Complexity and Education, 4(1), 7-24.

Sancho, J. M. \& Hernández, F. (2014) (Ed.). Maestros al Vaivén. Barcelona: Octaedro.

St. Pierre, E. (2016). The Empirical and the New Empiricisms. Cultural Studies Critical Methodologies, 16(2), 111-124.

St. Pierre, E.; Jackson, A. \& Mazzei, L. (2016). New Empiricisms and New Materialisms: Conditions for New Inquiry. Cultural Studies $\leftrightarrow$ Critical Methodologies, 16(2), 99-110. Ulmer, J. B. \& Koro-Ljungberg, M. (2015). Writing Visually Through (Methodological) Events and Cartography. Qualitative Inquiry, 21(2), 138-152. 